Z. klin. Chem. u. klin. Biochem.

7. Jg., S. 551-552, September 1969

\title{
Renale Cadmiumausscheidung, Lebensalter und arterieller Blutdruck
}

\author{
Von D. Szadkowski, K.-H. Schaller und G. LehNert
}

Aus dem Institut für Arbeits- und Sozialmedizin der Universität Erlangen-Nürnberg (Direktor: Prof. Dr. H. Valentin)

(Eingegangen am 9. Mai 1969)

Um zu prüfen, ob beim Menschen Beziehungen zwischen renaler Cadmiumausscheidung und Lebensalter oder dem Blutdruck bestehen, wurden bei 169 beruflich nicht Cd(II)-belasteten Probanden Cadmiumbestimmungen im Harn durchgeführt. Dabei zeigte sich eine mittlere Cd(II)-Ausscheidung von 1,25 $\mu \mathrm{g} / \mathrm{g}$ Kreatinin $(\mathrm{s}=1,02)$, der eine obere Normgrenze von $3,2 \mu \mathrm{g}$ Cd(II)/g Kreatinin entspricht. Durch Berechnung einer partiellen Regression bzw. Korrelation konnte eine Altersabhängigkeit der Cadmiumausscheidung statistisch gesichert werden. Dieser Einfluß ist für praktische Belange jedoch bedeutungslos. Eine Abhängigkeit des arteriellen Blutdrucks von der Cd(II)-Ausscheidungsrate ließ sich statistisch nicht sichern.

\section{The relationship between renal cadmium excretion, age, and arterial blood pressure}

In order to test for a relationship between renal cadmium excretion, age or blood pressure, cadmium was measured in the urine of 169 probands who were not occupationally exposed to $\mathrm{Cd}$ (II). The average Cd(II) excretion was $1.25 \mu \mathrm{g} / \mathrm{g}$ creatinine ( $\mathrm{s}=1.02)$, corresponding to a normal upper limit of $3.2 \mu \mathrm{g} \mathrm{Cd}(\mathrm{II}) / \mathrm{g}$ creatinine. A partial regression or correlation showed a statistically valid dependence of cadmium excretion on age. This effect is, however, of no practical importance. There was no statistically significant correlation between arterial blood pressure and $\mathrm{Cd}(\mathrm{II})$ excretion.

Seit den Arbeiten von Perry (1) und Schroeder (2) werden Zusammenhänge $z$ wischen dem Cadmiumgehalt der Nieren und einem arteriellen Bluthochdruck diskutiert. Als pathogenetisches Moment der Cadmiuminduzierten Hypertonie könnten die von FrIBERG (3) und BAADER (4) pathologisch-anatomisch nachgewiesenen Tubulusveränderungen und Schäden an den Glomerula gelten.

Tierexperimentell konnte durch intraperitoneale Injektion oder orale Applikation geringer Cadmiummengen ein Hochdruck erzeugt werden. Bei der Untersuchung menschlicher Organe auf Spurenelemente fanden sich im Nierenparenchym von Hypertonikern erhöhte Cadmium-Konzentrationen bzw. erhöhte Cadmium-Zink-Quotienten. Hypertoniker schieden im Harn bis zu $40 \mathrm{mal} \mathrm{mehr} \mathrm{Cd}$ (II) aus als gesunde Vergleichspersonen (2, 5-11). Schließlich fand sich bei epidemiologischen Studien, daß ein exhöhter Cadmiumgehalt in der Luft verschiedener amerikanischer Städte mit einer signifikanten Erhöhung der Todesrate an kardiovaskulären Erktankungen einherging (12).

Tipton (13), Schroeder (2) und Geldmacher-v. MallinCKRODt (14) nehmen auf Grund ihrer Untersuchungen eine Akkumulation von Cadmium im Nierengewebe mit fortschreitendem Lebensalter an. Es ist jedoch nichts darüber bekannt, ob die Cadmiumausscheidung im Harn sich in Abhängigkeit vom Alter ändert oder geschlechtsspezifische Unterschiede aufweist.
In der vorliegenden Arbeit sollte überprüft werden, inwieweit sich beim Menschen derartige Beziehungen zwischen renaler $\mathrm{Cd}(\mathrm{II})$-Ausscheidung und Lebensalter sichern lassen und ob Relationen zum systolischen oder diastolischen Blutdruck bestehen.

\section{Methodik}

Untersucht wurden 169 beruflich nicht cadmiumbelastete Probanden $\left(n+=64 ; n \sigma^{n}=105\right)$. Ihre Altersverteilung ist aus $A b$ bildung 1 ersichtlich.

Die $C d(I I)$-Bestimmungen im Harn erfolgten nach der von uns früher beschriebenen Methode mit atomabsorptionsspektrometrischer Endpunktmessung (15) aus Urinproben. Gleichzeitig wurde die Kreatininkonzentration im Harn bestimmt und die pro $1 \mathrm{~g}$ Kreatinin ausgeschiedene Cadmiummenge berechnet. Eine derartige Standardisierung hat sich uns bei Untersuchungen über die Ausscheidungsraten zahlreicher Stoffe, u. a. auch von Cadmium (16), gut bewährt.

Die Blutdruckmessungen erfolgten nach Riva-RoccI im Rahmen einer klinischen Untersuchung.

Statistisch wurden die gewonnenen Ergebnisse regressions- bzw. korrelationsanalytisch aufgearbeitet.

\section{Ergebnisse}

Die aus den Einzeldaten errechneten Mittelwerte und Standardabweichungen vonAlter, Cadmiumausscheidung sowie von systolischem und diastolischem Blutdruck sind in Tabelle 1 zusammengestellt worden.

Tab. 1

Mittelwerte und Standardabweichungen von Alter, Cadmiumausscheidung im Harn und Blutdruck für das Gesamtkollektiv sowie getrennt für Männer und Frauen

\begin{tabular}{|c|c|c|c|c|c|c|}
\hline & \multicolumn{2}{|c|}{$\begin{array}{l}\text { Gesamtkollektiv } \\
(n=169)\end{array}$} & \multicolumn{2}{|c|}{$\begin{array}{c}\text { Männer } \\
(\mathrm{n}=105)\end{array}$} & \multicolumn{2}{|c|}{$\begin{array}{l}\text { Frauen } \\
(\mathrm{n}=64)\end{array}$} \\
\hline & $\overline{\mathbf{x}}$. & s & $\overline{\mathbf{x}}$ & s & $\overline{\mathbf{x}}$ & $\mathbf{s}$ \\
\hline Alter [Jahre] & 38,4 & 24,2 & 35,7 & 23,4 & 42,8 & 25,0 \\
\hline Cd (II) $[\mu \mathrm{g} / \mathrm{g}$ Kreatinin] & 1,25 & 1,02 & 1,23 & 0,97 & 1,26 & 1,13 \\
\hline RR & 136,6 & 30,4 & 131,3 & 23,7 & 145,3 & 37,6 \\
\hline RRaiast. [mm Hg] & 82,6 & 15,9 & 81,5 & 14,0 & 84,4 & 18,6 \\
\hline
\end{tabular}


Tab. 2

Ergebnisse einer einfachen Regressionș- und Korrelationsanalyse der Werte des Gesamtkollektivs $(n=169)$

\begin{tabular}{|c|c|c|c|}
\hline$x \searrow^{y}$ & $\begin{array}{l}\text { RRsyst. } \\
\text { [mm Hg] }\end{array}$ & $\begin{array}{l}\text { RRdiast. } \\
\text { [mm Hg] }\end{array}$ & $\begin{array}{c}\text { Cd (II) } \\
{\left[\mu \mathrm{g} / \mathrm{g}^{\prime} \text { Kreatinin] }\right.}\end{array}$ \\
\hline $\begin{array}{l}\text { Alter } \\
\text { [Jahre] }\end{array}$ & $\begin{array}{l}y^{\prime}=102,5+0,89 x \\
r=0,7067 * * *\end{array}$ & $\begin{array}{l}y^{\prime}=78,3+0,12 x \\
r=0,3128 * * *\end{array}$ & $\begin{array}{l}y^{\prime}=0,92+0,009 x \\
\mathbf{r}=0,2009^{* *}\end{array}$ \\
\hline $\begin{array}{l}\mathrm{Cd}(\mathrm{II}) \\
{[\mu \mathrm{g} / \mathrm{g} \text { Kreatinin] }}\end{array}$ & $\begin{aligned} y^{\prime} & =132,0+3,73 x \\
& =0,1263\end{aligned}$ & $\begin{array}{l}y^{\prime}=80,7+1,54 x \\
r=0,0997\end{array}$ & - \\
\hline
\end{tabular}

\section{Diskussion}

Aus den vorliegenden Ergebnissen ist die obere Normgrenze der kreatininbezogenen Cd(II)-Ausscheidung im Harn als 95\%-Toleranzgrenze ohne Vertrauenswahrscheinlichkeit (17) errechnet worden. Danach sind Werte über 3,2 $\mu \mathrm{g}$ Cd(II)/g Kreatinin nicht mehr als normal zu bezeichnen. Dabei brauchen geschlechtsspezifische Unterschiede der Cadmium-Eliminationsraten nicht berücksichtigt zu werden (Tab. 1, $\mathrm{t}=-0,1831$ ).

Setzt man korrelationsanalytisch die Cd(II)-Ausscheidung im Harn zum jeweiligen Lebensalter der Probanden in Beziehung, so läßt sich eine Altersabhängigkeit statistisch sichern $(r=0,2009 * *)$. Demnach wird hier eine analoge Beziehung gefunden, wie sie andere Autoren $(13,2,14)$ für eine Abhängigkeit zwischen Lebensalter und Cadmiumgehalt des Nierengewebes beschrieben haben. SChroeder (2) wies aber andererseits - ebenso wie Perry (1) - auf Zusammenhänge zwischen dem Cadmiumgehalt der Nieren und einem arteriellen Bluthochdruck hin. Ferner ist bekannt, daß der arterielle Blutdruck mit zunehmendem Lebensalter ansteigt, wie wir auch an dem vorliegenden Kollektiv sichern konnten (Tab. 2).

Für eine exakte Aussage über die oben beschriebene Altersabhängigkeit der Cadmiumausscheidung müssen daher eventuelle Beziehungen zwischen dieser und dem Blutdruck berücksichtigt werden. Dies kann durch Berechnung des partiellen Korrelationskoeffizienten geschehen. Unter Eliminierung der systolischen bzw. diastolischen Blutdruckwerte ergibt sich dann eine Beziehung zwischen Lebensalter und Cadmiumausscheidung im Harn, die mit $r_{\text {part }}=0,1590 *$ bzw. $r_{\text {part }}=0,1795^{*}$ statistisch gesichert ist. $\mathrm{Da}$ sowohl bei der Ausschaltung des systolischen als auch des diastolischen Blutdruckes diese Beziehung signifikant ist, können für die Schätzung der entsprechenden partiellen Regressionsfunktion die Werte der mittleren arteriellen Blutdrucke für die Elimi- nation herangezogen werden. Es ergibt sich dann die Funktion $\mathrm{y}^{\prime}=1,148+0,0045 \times$ (Abb. 1). Der Verlauf der Regressionsgeraden läßt erkennen, daß die Cadmiumausscheidung in Abhängigkeit vom Alter nur geringfügig zunimmt.

Berechnet man in analoger Weise unter Ausschaltung des Alterseinflusses die Abhängigkeit des systolischen bzw. diastolischen Blutdruckes von der Höhe der Cadmiumausscheidung, so läßt sie sich mit $r_{\text {part }}=$ $-0,0225$ bzw. $r_{\text {part }}=0,0396$ nicht sichern. Dieses Ergebnis zeigt keine Analogie zu den von SCHROEDER (2) gefundenen Zusammenhängen zwischen Blutdruckhöhe und Cadmiumgehalt des Nierengewebes.

Auf Grund der vorliegenden Ergebnisse kann für die Interpretation der Resultate entsprechender ScreeningTests bei beruflich Cadmium-Exponierten (16) die zwar statistisch gesicherte, aber in der Auswirkung nur geringfügige Altersabhängigkeit der Cadmiumausscheidung im Harn vernachlässigt werden.



Abb. 1

Punkte-Korrelationsdiagramm zwischen Lebensalter und CadmiumAusscheidung im Harn bei 169 beruflich nicht Cd(II)-belasteten Probanden mit Angabe der partiellen Regression unter AusschluB des Blutdrucks (vgl. Text)

$\circ=O(n=64) \cdot=\sigma^{\prime \prime}(n=105) \quad y^{\prime} \odot O^{\prime \prime}=1,15+0,0045 x$

$\mathrm{r}^{*}: \mathrm{p}<0,05 . \quad \mathrm{r}^{* *}: \mathrm{p}<0,01$

\section{Literatur}

1. Perry, H. M. und H. A. Schroeder, J. Laborat. Clin. Med. S. Louis 46, 936 (1955). - 2. SChroeder, H. A., und J. BALASSA, J. Chron. Dis. 14, 236 (1961). - 3. Friberg, L., Acta med. Scand. 138, 1 (1950). - 4. BAAder, E. W., Dtsch. Med. Wschr. 76, 484 (1951). - 5. Schroeder, H. A., J. Amer. Med. Ass. 187, 358 (1964). 6. Schroeder, H. A., J. Chron. Dis. 18, 647 (1965). - 7. SchroeDER, H.A., S. S. Kroli, J. W. Littre, P. O. Livingston und M. A. G. Myres, Arch. Environ. Health 13, 788 (1966). - 8. Schroeder, H. A., Circulation 35, 570 (1967). - 9. Schroeder, H. A. und J. Buckman, Arch. Environ. Health 14, 693 (1967). 10. Schroeder, H. A., A. P. NAson, J. H. Tipton und J. J.
Balassa, J. chron. Dis. 20, 179 (1967). - 11. Schroeder, H. A., A. P. Nason, R. E. Prior, J. B. Reed und W. T. Haessler, Amer. J. Physiol. 214, 469 (1968). - 12. Carrol, R. E., J. Amer. Med. Ass. 198, 267 (1966). - 13. Trpron, J. H., Metal-Binding in Medicine, J. B. Lippincott Co., Philadelphia (1960). - 14. GeLDMacher-v. Mallinckrodt, $M$. und O. OpITz, Arbeitsmed. Sozialmed. Arbeitshyg. 3, 276 (1968). - 15. LeHNERT, G., K.-H. SCHALLER und Th. HAAS, diese Z. 6, 174 (1968). - 16. LEHNERT, G., G. Klavis, K.-H. Schaller und Th. HaAs, Brit. J. Industr. Med. 26, 156 (1969). - 17. Documenta Geigy, Wissenschaftliche Tabellen, 7. Auff., Geigy, Basel (1968). 852 Erlangen, Schillerstr. 25 